\title{
Optimization of Tungsten $\beta$-Phase Window for Spin-Orbit-Torque Magnetic Random-Access Memory
}

\author{
Kiran Kumar Vudya Sethu, ${ }^{1,2, *}$ Sambit Ghosh, ${ }^{1}$ Sebastien Couet, ${ }^{1}$ Johan Swerts, ${ }^{1}$ Bart Sorée, $, 1,2,3$ \\ Jo De Boeck, ${ }^{1,2}$ Gouri Sankar Kar, ${ }^{1}$ and Kevin Garello ${ }^{1,4, \dagger}$ \\ ${ }^{1}$ imec, Kapeldreef 75, Leuven 3001, Belgium \\ ${ }^{2}$ KU Leuven, Department of Electrical Engineering, Kasteelpark Arenberg 10, Leuven 3001, Belgium \\ ${ }^{3}$ University of Antwerp, Physics Department, Groenenborgerlaan 171, Antwerpen B-2020, Belgium \\ ${ }^{4}$ Université Grenoble Alpes, CEA, CNRS, Grenoble INP, SPINTEC, 38054 Grenoble, France
}

(Received 16 September 2021; accepted 4 November 2021; published 3 December 2021; corrected 20 December 2021)

\begin{abstract}
Switching induced by spin-orbit torque (SOT) is being vigorously explored, as it allows the control of magnetization using an in-plane current, which enables a three-terminal magnetic-tunnel-junction geometry with isolated read and write paths. This significantly improves the device endurance and the read stability, and allows reliable subnanosecond switching. Tungsten in the $\beta$ phase, $\beta-\mathrm{W}$, has the largest reported antidamping SOT charge-to-spin conversion ratio $\left(\theta_{\mathrm{AD}} \approx-60 \%\right)$ for heavy metals. However, $\beta$-W has a limitation when one is aiming for reliable technology integration: the $\beta$ phase is limited to a thickness of a few nanometers and enters the $\alpha$ phase above $4 \mathrm{~nm}$ in our samples when industryrelevant deposition tools are used. Here, we report our approach to extending the range of $\beta$-W, while simultaneously improving the SOT efficiency by introducing $\mathrm{N}$ and $\mathrm{O}$ doping of $\mathrm{W}$. Resistivity and XRD measurements confirm the extension of the $\beta$ phase from $4 \mathrm{~nm}$ to more than $10 \mathrm{~nm}$, and transport characterization shows an effective SOT efficiency larger than $-44.4 \%$ (reaching approximately $-60 \%$ for the bulk contribution). In addition, we demonstrate the possibility of controlling and enhancing the perpendicular magnetic anisotropy of a storage layer (Co-Fe-B). Further, we integrate the optimized W(O, N) into SOT magnetic random-access memory (SOT-MRAM) devices and project that, for the same thickness of SOT material, the switching current decreases by $25 \%$ in optimized $\mathrm{W}(\mathrm{O}, \mathrm{N})$ compared with our standard W. Our results open the path to using and further optimizing W for integration of SOT-MRAM technology.
\end{abstract}

DOI: 10.1103/PhysRevApplied.16.064009

\section{INTRODUCTION}

Current magnetic random-access memory (MRAM) technology uses spin transfer torque (STT) to switch the magnetization direction of the storage layer in a magnetic tunnel junction (MTJ). As an alternative, spin-orbit torque (SOT) [1], a recent development in the field of spintronics, has attracted considerable attention as a method to induce reversal of magnetization in nanomagnets $[2,3]$. The in-plane current required to generate a SOT allows one to write a magnetization state without passing a current through the tunnel barrier, unlike the case of STT, giving birth to the three-terminal SOT-MRAM concept [4], where the read operation is still performed by measuring the tunnel magnetoresistance (TMR). As such, the decoupling of the write and read current paths overcomes the main issues associated with STT-MRAM technology by preventing

\footnotetext{
*kiran.kumar.vudyasethu@imec.be

†kevin.garello@cea.fr
}

write errors during read operations, by eliminating the risk of voltage breakdown of the $\mathrm{MgO}$ tunnel barrier during write operations, and, importantly, by giving access to subnanosecond switching speeds and better endurance [5]. These promising characteristics have triggered strong interest from industry, and recent years have seen important efforts from research and development institutes to develop SOT-MRAM technology. In fact, SOT-MTJ integration on large-scale 200- and 300-mm wafers using CMOS-compatible processes has recently been demonstrated for both in-plane magnetized MTJs (IMTJs) [68] and perpendicularly magnetized MTJs (PMTJs) [1,9] (PMTJs being preferred by industry due to their scaling advantages). This opens up promising solutions not only for applications in cache memories [10,11], but also for other applications such as racetrack memories [12], logic $[13,14]$, and neuromorphic computing for ultralow-power computation [15-17].

Despite reports of the high performance and low power consumption of SOT PMTJs, numerous challenges need 
to be resolved before real applications can be envisaged. The first aspect relates to processing, manufacturing, and application compatibility, such as device yield [6,9], MTJ performance (TMR, retention), and, importantly, the need to sustain the back-end-of-line thermal budget $\left(400^{\circ} \mathrm{C}\right)$, where intermixing can severely affect the SOT efficiency [18]. Secondly, switching a perpendicular magnetization by SOT requires a static in-plane magnetic field to break the symmetry of the system and ensure deterministic reversal. As such a field is detrimental in memory applications, various credible approaches have been proposed to achieve field-free SOT switching, including exchange coupling to an antiferromagnet $[19,20]$, Ruderman-Kittel-KasuyaYosida coupling to a reference ferromagnet [21], geometrical asymmetry [22], STT assistance [23], and embedding a permanent magnet in the hard mask used to pattern the SOT track [24], each solution having pros and cons. Finally, these days the most critical aspect refers to the SOT charge-to-spin efficiency $\xi_{\text {SOT }}$ and the total current required to operate the device. The typical current densities reported are greater than $100 \mathrm{MA} \mathrm{cm}^{-2}$ for subnanosecond switching, translating to currents larger than $300 \mu \mathrm{A}$ in scaled SOT-MTJ cells $[10,25]$. Consequently, this requires increasing the size of the control transistors, directly affecting the cell footprint and density. It is therefore crucial to decrease the writing current to compete with existing technologies.

A simple expression for the threshold current density for switching is given by [26]

$$
J_{C, \text { perp }}^{\mathrm{SHE}}=\frac{e}{\hbar} \frac{M_{s} t_{\mathrm{FM}}}{\xi_{J}^{\mathrm{AD}}}\left(H_{K}^{\mathrm{eff}}-\sqrt{2} H_{x}\right),
$$

where $\xi_{J}^{\mathrm{AD}}$ is the antidamping SOT efficiency and $M_{s}$ is the saturation magnetization of the free layer, which is of thickness $t_{\mathrm{FM}}$ and has an effective perpendicular magnetic anisotropy field of $H_{K}^{\text {eff }}$. Optimizing the SOT efficiency is a straightforward way to reduce the threshold current density for switching to meet the requirements of industry. The main effects leading to spin-orbit torques are the spin Hall effect (SHE) and the Rashba effect $[1,27,28]$. Although the bulk or interface origin of the spin current giving rise to the antidamping (AD) and fieldlike (FL) effects is a matter of debate, it has been established that the strongest SOTs are found in normal metal with a large spin-orbit coupling such as the $5 d$ metals Pt, W, and Ta [1]. The SOT efficiency in these materials reaches up to $30 \%$, and in alloys and multilayers based on these metals shows the possibility to reach $80 \%$ [29], although no or only weak perpendicular magnetic anisotropy (PMA) of the free layer was reported. New exotic materials, such as topological insulators [30], have been reported to show even larger values (greater than $100 \%$ ) [31] but, to date, it is extremely complex to combine them with pMTJs.
Among the heavy metals, $\mathrm{W}$ in the $\beta$ phase has the highest antidamping SOT efficiency reported [32-36] $\left(\xi_{J}^{\mathrm{AD}} \approx\right.$ $-30 \%$ to $-60 \%$ ), and it is very compatible with PMTJ development [10]. However, following our recent development of tungsten-based SOT MTJs on large-scale wafers, we have reported that $\mathrm{W}$ loses its $\beta$ phase above $4 \mathrm{~nm}$ [10]. Such thin layers impose severe constraints on the choice of thickness and on the etching conditions, possibly leading to a strong distribution of the SOT resistance and efficiency from device to device. We report in this paper the possibility of optimizing the growth of $\beta$-W using dopants such as nitrogen and oxygen [37-39]. We develop a process in which growing a bilayer of oxygen- and nitrogendoped tungsten, $\mathrm{W}(\mathrm{O}, \mathrm{N})$, results in process control of the tungsten $\beta$ phase to more than $10 \mathrm{~nm}$, while concurrently improving the PMA as well as improving the SOT efficiency to $-44.4 \%$. Additionally, we show that implementing such an approach in scaled SOT-MTJs results in an effective reduction of the threshold current for switching by $25 \%$, indicating pathways to further engineering and optimizing SOT-MRAM technology.

\section{SAMPLE PREPARATION AND CHARACTERIZATION}

We use a Canon Anelva EC7800 cluster to sputter deposit our stacks on 300-mm Si wafers. The general layout of the stack is as follows: $\mathrm{Si} / \mathrm{SiO}_{2} / \mathrm{W}\left(t_{\mathrm{SOT}}\right) / \mathrm{Co}-\mathrm{Fe}-$ $\mathrm{B}(1) / \mathrm{MgO}(1) / \mathrm{TaO}_{x}(3)$, where the thicknesses are in nanometers and $t_{\mathrm{SOT}}$ is the thickness of the processed $\mathrm{W}$ layer. The $\mathrm{W}$ layer is deposited as a wedge. Note that the $\mathrm{SiO}_{2}$ substrate seed is grown by a PECVD method and flattened by chemical-mechanical polishing, as done during our device integration process. We observe that the nature of the oxide and its processing have an impact on the stack growth and the properties of the free layer, and hence it is important to develop solutions compatible with device fabrication methods. All sample stacks are annealed after deposition at $300^{\circ} \mathrm{C}$ for $30 \mathrm{~min}$.

We conduct the study using four types of samples, as sketched in Fig. 1(a). The major difference between the samples is the sputter-deposition conditions of the tungsten. Sample 1 (S1) consists of standard nondoped tungsten, W. We prepare sample 2 (S2) by first depositing a layer of $\mathrm{W}$, followed by oxygen flow for $300 \mathrm{~s}$ and subsequent deposition of an additional $\mathrm{W}$ layer to get a "laminate" of $\mathrm{W}$ with oxygen, resulting in an effective $\mathrm{W}(\mathrm{O})$ material. In sample $3(\mathrm{~S} 3), \mathrm{W}$ is reactively doped with nitrogen using an $\mathrm{Ar}: \mathrm{N}$ ratio of 10:1, to get $\mathrm{W}(\mathrm{N})$. Finally, in sample $4(\mathrm{~S} 4)$, we combine the deposition steps of the $\mathrm{W}(\mathrm{O})$ and $\mathrm{W}(\mathrm{N})$ samples to create a $\mathrm{W}(\mathrm{O}, \mathrm{N})$ bilayer. After deposition and annealing of the sample stacks, the sheet resistance is measured as a function of the SOT-layer thickness $\left(t_{\mathrm{SOT}}\right)$, and the saturation magnetization $\left(M_{s}\right)$ is evaluated using vibrating sample 
(a)

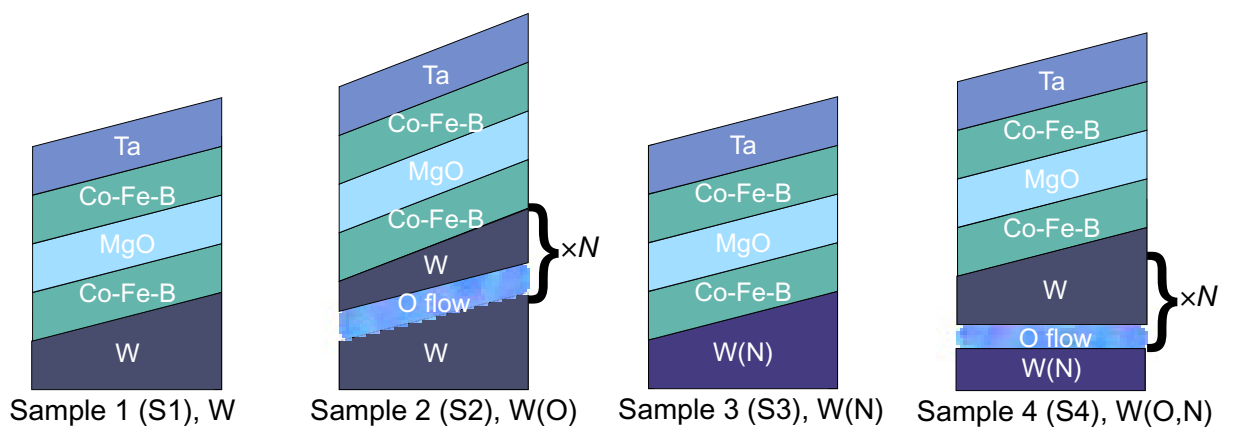

(b)

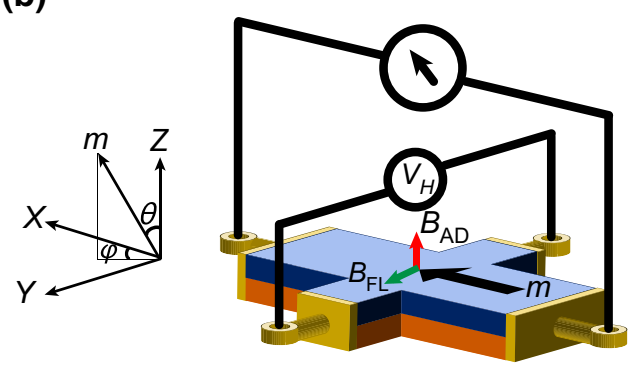

(c)

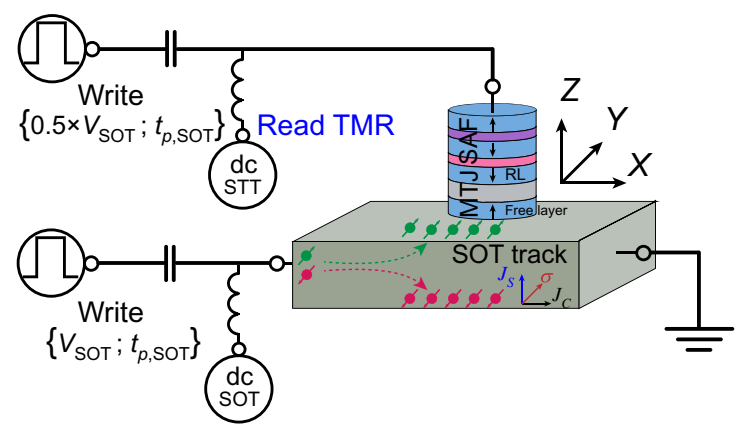

FIG. 1. (a) Sample stacks prepared for this study. The main difference between the samples is in the tungsten layer. Sample 1 (S1) is deposited with nondoped $\mathrm{W}$. In sample 2 (S2), the $\mathrm{W}$ layer is doped with oxygen. This is denoted as $\mathrm{W}(\mathrm{O})$. This layer is grown as nanolaminate repeats. The $\mathrm{W}$ layer in sample 3 (S3) is grown by reactive doping with nitrogen. This is denoted as $\mathrm{W}(\mathrm{N})$. In sample $4(\mathrm{~S} 4)$, the $\mathrm{W}$ is prepared by doping with both nitrogen and oxygen. This is denoted as $\mathrm{W}(\mathrm{O}, \mathrm{N})$. The Co-Fe-B is capped with $\mathrm{MgO}(1 \mathrm{~nm})$, Co-Fe-B dusting, and Ta capping layers. Slanted layers indicate layers deposited as a wedge on a single wafer. (b) Schematic illustration showing Hall-bar measurement geometry along with definition of the coordinates used in the harmonic Hall-voltage measurements for the quantification of SOTs in our samples. (c) Schematic illustration of electrical measurements of SOT-MRAM cell. $V_{\text {SOT }}$ is a voltage pulse with a pulse width $t_{p \text {,SOT }}$, applied through the SOT track for electrical switching of the free layer of the MTJ. The orientation of the MTJ (parallel or antiparallel) is measured by applying a de voltage through the MTJ. dc TMR measurements are performed to measure the hysteresis loop of the free layer. SAF, synthetic anti-ferromagnet; RL, reference layer.

magnetometry. Subsequently, Hall-bar devices of width $w=10 \mu \mathrm{m}$ and length $L=100 \mu \mathrm{m}$ are patterned using photolithography and dry etched by Xe ion milling.

We make use of the harmonic Hall-voltage method [40-42] to quantify the magnetotransport coefficients, the magnetic anisotropy $B_{K}$, and the current-induced $\mathrm{AD}$ and FL effective SOT fields, $B_{\mathrm{AD}}$ and $B_{\mathrm{FL}}$, respectively. We use a low-frequency ac current of frequency $10 \mathrm{~Hz}$ and a current amplitude $I_{\text {total }}$, corresponding to a current density $J$ of $2 \mathrm{MA} \mathrm{cm}^{-2}$, calculated for the full metallic thickness, i.e., including the thickness of the Co-Fe-B layer. We use spherical coordinates, and the current direction is defined to be along the $X$ axis, as shown in Fig. 1(b). First-harmonic $\left(V_{1 \omega}\right)$ and second-harmonic $\left(V_{2 \omega}\right)$ signals are obtained simultaneously from a fast Fourier transform of the acquired longitudinal and transverse signals. These signals are measured as a function of the amplitude, $B_{\text {ext }}$, and angles, $\left[\theta_{B} ; \phi_{B}\right]$, of the external field. The sample resistance is estimated precisely from the longitudinal signals, while the transverse resistance $R_{\text {Hall }}$ is read from the planar Hall $\left(R_{\mathrm{PHE}}\right)$ and anomalous Hall $\left(R_{\mathrm{AHE}}\right)$ resistances: $R_{\text {Hall }}=R_{\mathrm{AHE}} \cos (\theta)+R_{\mathrm{PHE}} \sin ^{2}(\theta) \sin (2 \phi) . R_{\text {Hall }}$ is used to quantify the polar angle of the magnetization $(\theta)$ during the measurements, while $R_{\mathrm{AHE}}$ is extracted from an outof-plane field scan $\left(\theta_{B}=0^{\circ}\right)$, and $R_{\mathrm{PHE}}$ is obtained from an in-plane angular scan $\left(\theta_{B}=90^{\circ}\right)$ at a constant in-plane field of $B_{\text {ext }}=2 \mathrm{~T}$.

The effective perpendicular magnetic anisotropy $\left(B_{K}^{\text {eff }}\right)$ is estimated from the $R_{\text {Hall }}$ signal of a field-sweep measurement in which a magnetic field $B_{\text {ext }}$ is applied almost in-plane to the sample $\left[\theta_{B}=86^{\circ} ; \phi_{B}=0^{\circ}\right]$. A macrospin model [Eq. (2)] is used to evaluate $B_{K}^{\text {effr }}$ :

$$
\begin{aligned}
B_{K}^{\mathrm{eff}} & =B_{K}-B_{\mathrm{dem}}=\left[\frac{\cos \theta_{B}}{\cos \theta}-\frac{\sin \theta_{B}}{\sin \theta}\right] B_{\mathrm{ext}}, \\
B_{\mathrm{dem}}^{\mathrm{eff}} & =B_{\mathrm{dem}}-B_{K}=\mu_{0} M_{s}-\frac{2 K}{M_{s}},
\end{aligned}
$$

where the polar angle of the magnetization $\theta$ is estimated at every field step. Here, $K$ is the uniaxial anisotropy constant and $M_{s}$ is the saturation magnetization. When the interfacial magnetic anisotropy field $\left(B_{K}\right)$ dominates and exceeds the demagnetizing field $\left(B_{\mathrm{dem}}\right)$, the samples have a positive $B_{K}^{\text {eff }}$ and therefore PMA. Otherwise, the samples 
have in-plane magnetic anisotropy (IMA). In samples with IMA, the effective demagnetizing field $\left(B_{\text {dem }}^{\text {eff }}\right)$ in Eq. (3) is estimated as the out-of-plane field at which the transverse first-harmonic resistance saturates. To quantify the SOT effective fields, we follow a procedure adapted from Ref. [41], which takes into account thermal effects and allows quantification of SOT in both in-plane and out-ofplane magnetized systems. $B_{\mathrm{AD}}$ and $B_{\mathrm{FL}}$ are estimated from the following equations:

$$
\begin{aligned}
R_{2 \omega}^{\pi / 4} & =B_{\mathrm{AD}}^{\theta} \cos \frac{\pi}{4} \frac{R_{\mathrm{AHE}}}{B_{\mathrm{ext}}+B_{K}^{\mathrm{eff}}+B_{\mathrm{dem}}^{\mathrm{eff}}}+R_{\nabla T_{z}} \cos \frac{\pi}{4}, \\
R_{2 \omega}^{0} & =\frac{R_{2 \omega}^{\pi / 4}}{\cos (\pi / 4)}+\frac{-2 R_{\mathrm{PHE}}}{B_{\mathrm{ext}}} B_{\mathrm{FL}}^{\phi} .
\end{aligned}
$$

Here, $R_{2 \omega}^{\pi / 4}$ and $R_{2 \omega}^{0}$ correspond to the second-harmonic resistance measured by sweeping the in-plane field at $\left[\theta_{B}=90^{\circ} ; \phi_{B}=45^{\circ}\right]$ and $\left[\theta_{B}=90^{\circ} ; \phi_{B}=0^{\circ}\right]$, respectively. $R_{\nabla T_{z}}$ is the thermal resistance induced by out-ofplane thermal gradients [41].

In a second step, the effective antidamping (fieldlike) fields are converted into a charge-to-spin conversionefficiency parameter $\xi_{j}^{\mathrm{AD}(\mathrm{FL})}$ using

$$
\xi_{j}^{\mathrm{AD}(\mathrm{FL})}=\frac{2 e}{\hbar} \frac{M_{s}}{J} t_{\mathrm{FM}} B_{\mathrm{AD}(\mathrm{FL})},
$$

where $e$ is the electronic charge, $\hbar$ is the reduced Planck constant, and $t_{\mathrm{FM}}$ is the thickness of the ferromagnet.

We report on the electrical switching properties of threeterminal PMTJ SOT-MRAM devices in Sec VI. The MTJ stack for the results presented in Sec.VI is as follows: $\mathrm{Si} / \mathrm{SiO}_{2} /[\mathrm{W}(3.5)$ or $\mathrm{W}(\mathrm{O}, \mathrm{N})(6)] / \mathrm{Co}-\mathrm{Fe}-\mathrm{B}(0.9) / \mathrm{MgO}(1) /$ $\mathrm{Co}-\mathrm{Fe}-\mathrm{B}(1) / \mathrm{W}(0.3) / \mathrm{SAF}(10.5)$ (with thicknesses in nanometers). Further details of the stack and device fabrication process can be found in Refs. [10,24,25]. To determine the effective perpendicular magnetic anisotropy $\left(B_{K}^{\text {eff }}\right)$ and the retention $(\Delta)$ of the PMTJ free layer, the procedure used is as follows: hysteresis loops of the free layer are measured 600 times, from which the switching-field probability distribution, $P_{\mathrm{sw}}\left(B_{s}\right)$, is evaluated. Equation (7) is used to fit the switching-field probability distribution and extract $B_{K}^{\text {eff }}$ and $\Delta$ of the free layer as free parameters, as reported in Ref. [43]:

$$
\begin{aligned}
P_{\mathrm{sw}}\left(B_{s}\right)= & 1-\exp \left\{\frac{-B_{K}^{\mathrm{eff}} f_{0}(\sqrt{\pi} / 2)}{R \sqrt{\Delta}}\right. \\
& \left.\times \operatorname{erfc}\left[\sqrt{\Delta}\left(1-\frac{B_{s}}{B_{K}^{\mathrm{eff}}}\right)\right]\right\},
\end{aligned}
$$

where $R$ is the sweep rate of the magnetic field and $f_{0}$ is the attempt frequency. A schematic illustration of the measurements of the electrical switching of the free layer by
SOT presented in this paper is shown in Fig. 1(c). A voltage pulse of amplitude $V_{\text {SOT }}$ and width $t_{P \text {, SOT }}$ is applied through the SOT track to write the free layer. To avoid a STT contribution, we compensate the potential drop underneath the free layer by applying $V_{\mathrm{STT}}\left(t_{P, \mathrm{STT}}\right)=0.5 \times$ $\mathrm{V}_{\mathrm{SOT}}\left(t_{P, \mathrm{SOT}}\right)$ across the MTJ in synchronization with the SOT write pulse [25]. A dc bias $V_{\text {read }}=10 \mathrm{mV}$ through the MTJ is used to measure the TMR.

\section{EXTENSION OF TUNGSTEN $\beta$ PHASE WINDOW}

Thin films of $\mathrm{W}$ can exist in two crystalline phases $[32,34,36,37,44,45]$ : an $\alpha$ phase $(\alpha-\mathrm{W})$, which has a low resistivity $\left(5-20 \mu \Omega \mathrm{cm}\right.$ ) and low $\xi_{J}^{\mathrm{AD}}$ (note that $\alpha-\mathrm{W}$ is widely used in industry for contact metallization), and a $\beta$ phase $(\beta-\mathrm{W})$, with a larger resistivity $(100-300 \mu \Omega \mathrm{cm})$ and, importantly, a very large $\xi_{J}^{\mathrm{AD}}$. Therefore, to check for the presence of the $\beta$ phase in different samples, we measure the sheet resistance by a four-point-probe technique as a function of the thickness $t_{\text {SOT }}$ of the $\mathrm{W}, \mathrm{W}(\mathrm{N}), \mathrm{W}(\mathrm{O})$, and $\mathrm{W}(\mathrm{O}, \mathrm{N})$ samples. The resistivity of the samples calculated from the sheet resistance is shown in Fig. 2(a). The resistivity shown here is the total resistivity of the SOT/free-layer bilayer, and therefore it also includes the resistivity of the Co-Fe-B layer. Clearly, the resistivity of the standard tungsten sample $\mathrm{S} 1$ (black curve) decreases significantly beyond $4 \mathrm{~nm}$, indicating a $\beta$-to- $\alpha$ phase transition $\left(t_{\beta \text {-to- } \alpha}\right)$ of $\mathrm{W}$ and an $\alpha$ phase from approximately $5 \mathrm{~nm}$ and above. The resistivity of $\alpha-\mathrm{W}(25 \mu \Omega \mathrm{cm})$ is approximately 5 times smaller than that of $\beta-\mathrm{W}$. In the $\mathrm{W}$ samples (S1), the resistivity of the $\beta-\mathrm{W}, \rho_{\beta}$-W, is $140 \mu \Omega \mathrm{cm}$, which is in the
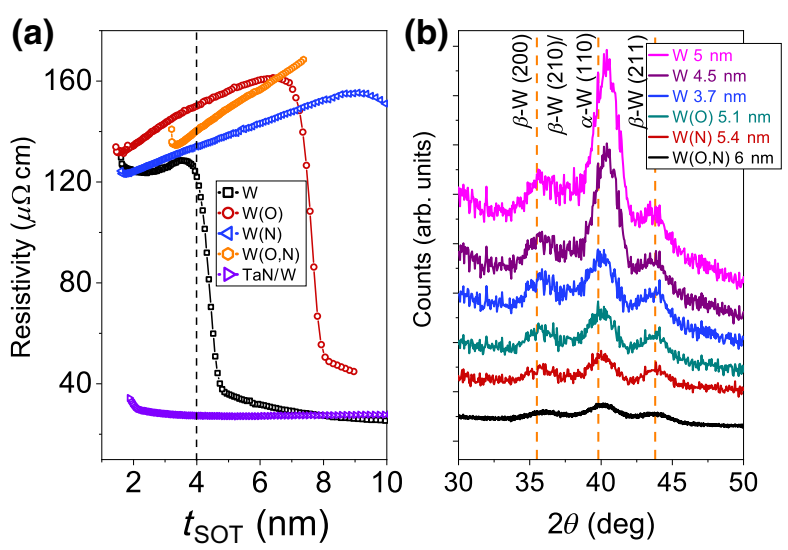

FIG. 2. (a) Sample resistivity $\rho$ estimated from four-pointprobe sheet-resistance measurements as a function of $\mathrm{W}$ layer thickness. (b) Grazing-incidence XRD spectra of samples. The thickness of the $\mathrm{W}$ layer, along with the sample type, is indicated in the legend. Peak positions corresponding to different crystal phases of tungsten are indicated, with reference to the lattice plane. 
lower range of $\beta$-W resistivity values reported in the literature $[32,34]$. The presence of some $\alpha$ grains could lead to such moderate resistivity. Since $\mathrm{W}$ has bad adhesion to oxides, we also prepare samples including a 1-nm TaN seed layer to prevent delamination. Remarkably, TaN and other seeds tested, such as $\mathrm{Ti}$ and TiN, favor high-quality $\mathrm{W}$ growth in the $\alpha$ phase and prevent the formation of the $\beta$ phase above $1.5 \mathrm{~nm}$, as shown in Fig. 2(a) (purple curve), strongly limiting the use of seed and adhesion materials.

However, there are reports showing that the presence of oxygen or nitrogen adsorbed onto the surface provides nucleation sites for the growth of metastable $\beta$-W [37-39]. In the presence of a large number of such nucleation sites, $\beta$-W nucleates more readily than $\alpha-\mathrm{W}$, and thereby the $\beta$ phase becomes the majority phase, in addition to some $\alpha-\mathrm{W}$ grains. Furthermore, other reports show that doping $\mathrm{W}$ with oxygen increases the antidamping efficiency to as high as $-50 \%$ [46]. Therefore, taking account of this information, we prepare tungsten samples doped with $\mathrm{O}(\mathrm{S} 2)$ and $\mathrm{N}$ (S3), expecting to extend the $\beta$ phase and improve $\xi_{J}^{\mathrm{AD}}$.

As is apparent in Fig. 2(a) from the resistivity measurements, the $\beta$ phase is extended to thicker tungsten in samples $\mathrm{S} 2$ and $\mathrm{S} 3$ compared with $\mathrm{S} 1$. In the oxygen-doped sample ( $\mathrm{S} 2$, red curve), $\beta$-W exists up to $7 \mathrm{~nm}$ of tungsten, beyond which we see a transition into the $\alpha$ phase from the drop in resistivity, as in $\mathrm{S} 1 . \rho_{\beta-\mathrm{W}}$ in $\mathrm{W}(\mathrm{O})$ is $157 \mu \Omega \mathrm{cm}$. The $\beta$-phase window can be further increased by adjusting the $\mathrm{W}$ thickness and the lamination repeats. Furthermore, in samples doped with nitrogen (S3, blue curve), the $\beta$ phase persists above $10 \mathrm{~nm}$, with $\rho_{\beta \text {-W }} \approx 178 \mu \Omega \mathrm{cm}$. Although both doped tungsten samples have an extended $\beta$ phase, as we show in the next section, the $\mathrm{W}(\mathrm{O})$ samples possess PMA and the $\mathrm{W}(\mathrm{N})$ samples have IMA, while $\xi_{J}^{\mathrm{AD}}$ is larger in the W(N) samples (see Sec. V). For the development of PMTJ SOT MRAMs, PMA in the free layer is mandatory. Therefore, to make use of the best of both approaches, we prepare samples combining nitrogen and oxygen doping, $\mathrm{W}(\mathrm{O}, \mathrm{N})$ ( $\mathrm{S} 4$, orange data points), with thicknesses of 3.6 and $6 \mathrm{~nm}$. $\rho_{\beta \text {-W }}$ for $\mathrm{W}(\mathrm{O}, \mathrm{N})$ is found to be $200 \mu \Omega \mathrm{cm}$.

To confirm the presence of the $\beta$ phase in the blanket films, we perform grazing-incidence $X$-ray diffraction (GI$\mathrm{XRD)}$ measurements. Three signature peaks in the $2 \theta$ scan $\left(35.5^{\circ}, 39.9^{\circ}\right.$, and $\left.43.9^{\circ}\right)$ characterize $\beta$-W, while $\alpha-\mathrm{W}$ exhibits a single peak at $40.2^{\circ}$. When both $\beta$ and $\alpha$ tungsten coexist, such as in the $\beta$-to- $\alpha$ phase-transition region, peaks for both phases should appear in the XRD spectrum. Figure 2(b) shows GI-XRD spectra for a selection of thicknesses for $\mathrm{S} 1$ and for different $\mathrm{W}$ deposition processes in $\mathrm{S} 2$, S3, and $\mathrm{S} 4$, with $t_{\mathrm{SOT}}>4 \mathrm{~nm}$ (or $t_{\mathrm{SOT}}>t_{\beta \text {-to- } \alpha}$ for $\mathrm{S} 1)$. For S1, it is apparent that the central peak increases in intensity relative to the other two shoulder peaks, indicating that majority of the grains are $\alpha-\mathrm{W}$ for $t_{\mathrm{SOT}}>4$ $\mathrm{nm}$, as inferred from the resistivity measurement. $\operatorname{In} \mathrm{W}(\mathrm{N})$,
$\mathrm{W}(\mathrm{O})$, and $\mathrm{W}(\mathrm{O}, \mathrm{N})$, the three peaks corresponding to the $\beta$ phase are observed but with low amplitude, confirming our conclusion from the resistivity measurement.

\section{MAGNETIC PROPERTIES OF W/Co-Fe-B/MgO STACKS}

In this section, we first discuss the trends of the saturation magnetization $\mu_{0} M_{s}$ vs $\mathrm{t}_{\mathrm{SOT}}$, shown in Fig. 3(a). S1 - undoped tungsten samples - shows a constant value of $\mu_{0} M_{s}$ of $1.1 \mathrm{~T}$ (black curve), which degrades to 0.9 $\mathrm{T}$ when $t_{\mathrm{SOT}}<2.4 \mathrm{~nm}$. This is probably related to the interface roughness and quality, resulting in an increased Co-Fe-B dead layer. In comparison, S2 (red curve) shows lower values of $\mu_{0} M_{s}=0.9 \mathrm{~T}$, strongly degrading below $3.4 \mathrm{~nm}$, which indicates a sizable impact of oxygen on the Co-Fe-B, with partial oxidation of the Co-Fe-B layer. This influence of oxygen can be mitigated and optimized through the exposure time of the $\mathrm{W}$ to oxygen flow during growth, as we show later for the $\mathrm{W}(\mathrm{O}, \mathrm{N})$ samples. The saturation magnetization for $\mathrm{S} 3$ reaches substantially larger values of $1.2 \mathrm{~T}$ compared with previous samples and reaches $1.6 \mathrm{~T}$ for $t_{\mathrm{SOT}}>5 \mathrm{~nm}$. We attribute these large values to a quasiamorphous $\mathrm{W}$ phase induced by nitrogen, improving the interface roughness and favoring better Co-Fe-B growth, as commonly observed when a smooth $\mathrm{TaN}$ seed is used for MTJ growth [47]. Finally, in the $\mathrm{W}(\mathrm{O}, \mathrm{N})$ stacks, $\mu_{0} M_{s}$ reaches 1.02 and $1.17 \mathrm{~T}$ for $t_{\mathrm{SOT}}$ equal to 3.6 and $6 \mathrm{~nm}$, respectively.

We now turn to the impact of the doping process on the magnetic anisotropy. The magnetic anisotropy reported here is estimated in Hall-bar devices, as explained in Sec. II. Positive values indicate the presence of PMA $\left(B_{K}^{\text {eff }}\right)$,
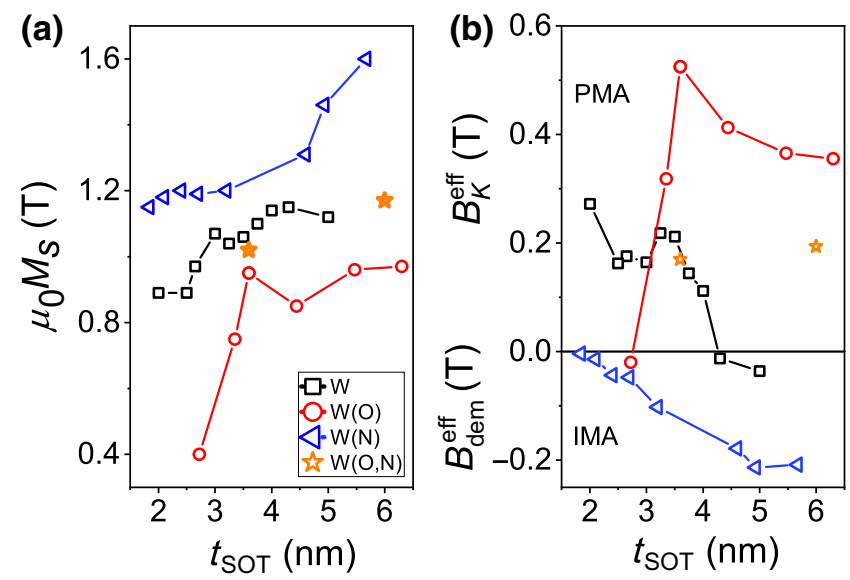

FIG. 3. (a) Saturation magnetization of Co-Fe-B as a function of tungsten layer thickness. Sample types are indicated in the legend. (b) Effective anisotropy field, $B_{K}^{\text {eff }}$, of Co-Fe-B vs W thickness measured at Hall-bar level as described in the text. Positive values of $B_{K}^{\text {eff }}$ indicate PMA, and negative values correspond to a demagnetization field and therefore IMA. 
and negative values represent IMA ( $B_{\mathrm{dem}}^{\text {eff }}$ ) in the Co-Fe-B. The magnetic anisotropy vs $t_{\mathrm{SOT}}$ is shown in Fig. 3(b). S1 shows a moderate PMA with $B_{K}^{\text {eff }} \approx 0.2 \mathrm{~T}$, with a PMAto-IMA transition above $4 \mathrm{~nm}$ of $\mathrm{W}$. This coincides with the $\beta$-to- $\alpha$ phase transition of $\mathrm{W}$ described in the previous section. When oxygen is introduced, as in S2 (red curve), we observe a significant increase in the PMA, with $B_{K}^{\text {eff }}$ reaching up to $0.6 \mathrm{~T}$. This is interpreted as an enhancement of the $\mathrm{W}(\mathrm{O}) / \mathrm{Co}-\mathrm{Fe}-\mathrm{B}$ interfacial anisotropy induced by oxygen [48]. This interface anisotropy is usually provided by a $\mathrm{Co}-\mathrm{Fe}-\mathrm{B} / \mathrm{MgO}$ interface, and here $\mathrm{W}(\mathrm{O})$ provides an additional way to increase the anisotropy and volume of the SOT-PMTJ, similarly to the dual-MgO approach used in standard STT-MRAM technology [49,50]. Below $t_{\mathrm{SOT}}=3.4 \mathrm{~nm}$, the anisotropy strongly decreases, and correlates with the trend in $\mu_{0} M_{s}$. We therefore conclude that the $\mathrm{Co}-\mathrm{Fe}-\mathrm{B}$ is overoxidized due to an excess of oxygen [51,52].

The nitrogen-doped samples S3 (blue curve) do not exhibit any PMA for the entire thickness range of W(N). The in-plane anisotropy increases with the thickness of $\mathrm{W}(\mathrm{N})$. Although the saturation magnetization does not vary much with the thickness until $3.5 \mathrm{~nm}$ of $\mathrm{W}(\mathrm{N})$ is reached, it does increase for thicknesses greater than 3.5 $\mathrm{nm}$. This increase in $\mu_{0} M_{s}$ causes the demagnetizing field to dominate over any weak interface anisotropy that might be present.

The importance of the role of oxygen in inducing PMA in Co-Fe-B is further elucidated by the manifestation of PMA in $\mathrm{W}(\mathrm{O}, \mathrm{N})$ samples with values of the effective anisotropy field in between those of the W and W(O) samples. $B_{K}^{\mathrm{eff}}$ is $0.4 \mathrm{~T}$ for the $3.6-\mathrm{nm}$ sample and $0.3 \mathrm{~T}$ for $t_{\text {SOT }}$ equal to $6 \mathrm{~nm}$, thus showing the possibility of controlling and enhancing the interfacial anisotropy through the W(O)/Co-Fe-B interface.

\section{QUANTIFICATION OF SPIN-ORBIT TORQUES}

Here we report on the influence of doping on the SOT efficiency. In-plane field-scan measurements at $\left[\theta_{B}=\right.$ $\left.90^{\circ} ; \phi_{B}=45^{\circ}\right]$ and $\left[\theta_{B}=90^{\circ} ; \phi_{B}=0^{\circ}\right]$ are performed to quantify the antidamping and fieldlike effective fields, respectively. From $B_{\mathrm{AD}}$ and $B_{\mathrm{FL}}$, the associated SOT efficiencies $\xi_{J}^{\mathrm{AD}}$ and $\xi_{J}^{\mathrm{FL}}$ are estimated as detailed in Sec. II. The trends in $\xi_{J}^{\mathrm{AD}}$ and $\xi_{J}^{\mathrm{FL}}$ vs $t_{\mathrm{SOT}}$ are summarized in Figs. 4(a) and 4(b), respectively.

$\xi_{J}^{\mathrm{AD}}$ reaches a maximum of $-35 \%$ in the $\mathrm{W}$ samples S1 (black curves), comparable to initial reports [32]. The antidamping SOT efficiency varies from $-19 \%$ to $-35 \%$ for thicknesses ranging from 2 to $4 \mathrm{~nm}$, respectively, indicating bulklike behavior of the spin Hall effect. As anticipated, $\xi_{J}^{\mathrm{AD}}$ drops considerably when the tungsten transforms to the $\alpha$ phase, and it is not detectable beyond 5 $\mathrm{nm} . \xi_{J}^{\mathrm{FL}}$ fluctuates around $-9 \%$ and drops rapidly to $-2 \%$ on the cusp of the transition to the $\alpha$ phase. Remarkably,
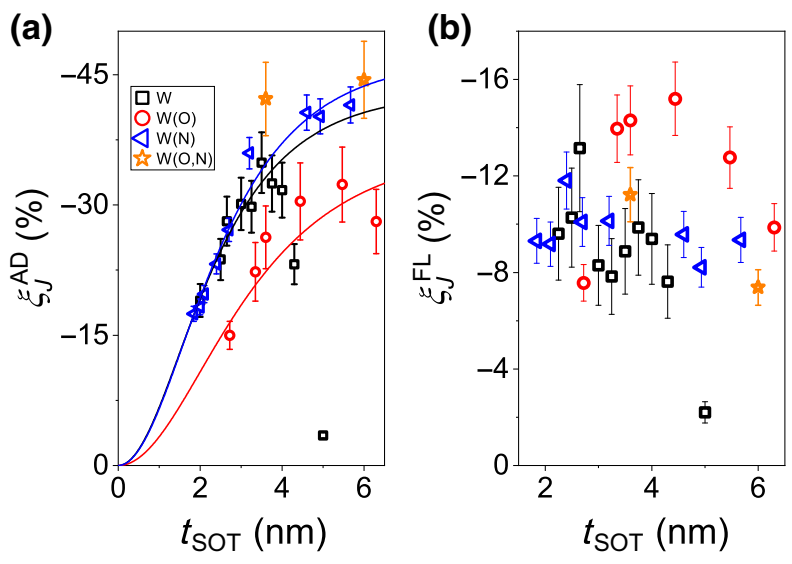

FIG. 4. (a) Antidamping SOT efficiency, $\xi_{J}^{\mathrm{AD}}$, of different types of $\mathrm{W}$ as a function of tungsten layer thickness. The solid lines are fits to the experimental data points using a drift-diffusion model to extract the intrinsic spin Hall angle $\theta_{\mathrm{SH}}^{\text {eff }}$ and the spin diffusion length $\lambda_{\text {sf. }}$. (b) Fieldlike SOT efficiency, $\xi_{J}^{\mathrm{FL}}$, as a function of SOT-layer thickness $t_{\mathrm{SOT}}$.

$\xi_{J}^{\mathrm{FL}}$ does not depend on the thickness of $\mathrm{W}$, indicating that its origin might arise from the Rashba effect present at the W/Co-Fe-B interface due to the high spin-orbit coupling.

Contrary to a report of efficiencies as high as $-50 \%$ in $\mathrm{W}(\mathrm{O})$ [46], we do not observe a real impact of oxygen on $\xi_{J}^{\mathrm{AD}}$ in $\mathrm{S} 2$, where $\xi_{J}^{\mathrm{AD}}$ reaches a maximum of $-32.3 \%$ at $5.5 \mathrm{~nm}$, comparable to that for S1. Moreover, in our samples, $\xi_{J}^{\mathrm{AD}}$ behaves as a bulklike effect, as opposed to an interfacial effect as in the earlier report [46]. This discrepancy may come from differences in the thin-film deposition process, since our $\mathrm{W}(\mathrm{O})$ samples are not prepared by reactive doping, in contrast to the samples reported in Ref. [46]. Additionally, the difference in efficiency could also be attributed to a possible variation of oxygen concentration as a function of $t_{\text {SOT }}$ in our $\mathrm{W}(\mathrm{O})$ films, differences in the measurement technique used to quantify the SOT, and formation of an inadvertent oxide layer at the interface, affecting the interface spin transmission [48]. The slight reduction in $\xi_{J}^{\mathrm{AD}}$ in the samples above $5 \mathrm{~nm}$ could be due to an increase in $\alpha$-tungsten grains relative to $\beta$ tungsten $[33,53]$. Interestingly, $\xi_{J}^{\mathrm{FL}}$ increases to $-15 \%$ and is highest compared with other samples, pointing to a possible role of oxygen at interfaces in the nature and amplitude of the FL efficiency [48].

Although there is no PMA here, we evaluate the SOT in the nitrogen-doped samples $\mathrm{S} 3$ (blue curves). $\xi_{J}^{\mathrm{AD}}$ for $\mathrm{W}(\mathrm{N})$ is higher compared with the $\mathrm{W}$ case, as visible in Fig. 4(a). It follows a bulk SHE model, and a maximum efficiency of $-41.5 \%$ is reached for $5.5 \mathrm{~nm}$ of $\mathrm{W}(\mathrm{N})$. This further confirms that the high resistivity of the thicker tungsten in these samples is due to the presence of $\beta-\mathrm{W}$. The larger $\xi_{J}^{\mathrm{AD}}$ is not straightforward to explain, but could be attributed to increased scattering due to a 
TABLE I. Resistivity of $\mathrm{W}\left(\rho_{\beta-\mathrm{W}}\right)$ and Co-Fe-B $\left(\rho_{\beta \text {-Co-Fe-B }}\right)$ calculated from the dependence of the Hall-bar resistance on the $\mathrm{W}$ thickness $\left(t_{\mathrm{SOT}}\right)$; spin diffusion length $\left(\lambda_{\mathrm{sf}}\right)$ and intrinsic spin Hall efficiency $\left[\theta_{\mathrm{SH}}^{\mathrm{eff}}(\%)\right]$ estimated from the total current flowing through the stack $\left(I_{\text {total }}\right)$ and from the current flowing in the SOT layer alone $\left(I_{\mathrm{SOT}}\right)$; and spin Hall conductivity $\left(\sigma_{\mathrm{SH}}\right)$, for samples $\mathrm{S} 1, \mathrm{~S} 2$, and S3.

\begin{tabular}{lcccccccc}
\hline \hline Sample & $\mathrm{W}$ type & $\begin{array}{c}\rho_{\beta-\mathrm{W}} \\
(\mu \Omega \mathrm{cm})\end{array}$ & $\begin{array}{c}\rho_{\mathrm{Co}-\mathrm{Fe}-\mathrm{B}} \\
(\mu \Omega \mathrm{cm})\end{array}$ & $\begin{array}{c}\theta_{\mathrm{SH}}^{\text {eff }}(\%) \\
\left(I_{\text {total }}\right)\end{array}$ & $\begin{array}{c}\lambda_{\mathrm{sf}}(\mathrm{nm}) \\
\left(I_{\text {total }}\right)\end{array}$ & $\begin{array}{c}\theta_{\mathrm{SH}}^{\text {eff }}(\%) \\
\left(I_{\mathrm{SOT}}\right)\end{array}$ & $\begin{array}{c}\lambda_{\text {sf }}(\mathrm{nm}) \\
\left(I_{\mathrm{SOT}}\right)\end{array}$ & $\begin{array}{c}\sigma_{\mathrm{SH}}\left[\mathrm{k}(\Omega \mathrm{m})^{-1}\right] \\
\left(I_{\mathrm{SOT}}\right)\end{array}$ \\
\hline $\mathrm{S} 1$ & $\mathrm{~W}$ & 138.7 & 76.4 & $-43 \pm 3.8$ & $1.7 \pm 0.2$ & $-54.4 \pm 3.1$ & $1.1 \pm 0.1$ & 3.9 \\
$\mathrm{~S} 2$ & $\mathrm{~W}(\mathrm{O})$ & 170.8 & 84.7 & $-36.6 \pm 5.6$ & $2.3 \pm 0.4$ & $-53.9 \pm 6.8$ & $2 \pm 0.3$ & 3.1 \\
$\mathrm{~S} 3$ & $\mathrm{~W}(\mathrm{~N})$ & 149.4 & 93.2 & $-46.9 \pm 2.2$ & $1.8 \pm 0.1$ & $-56.5 \pm 2.1$ & $1.2 \pm 0.1$ & 3.8 \\
\hline \hline
\end{tabular}

quasiamorphous phase, as presumed in Ref. [34]. It is also possible that better interface quality induced by amorphous growth improves the transparency of the interface to the spin and consequently maximizes the torque transferred to the ferromagnet. The fieldlike torque efficiency $\xi_{J}^{\mathrm{FL}}$ in these samples does not vary with thickness, and fluctuates around $-10 \%$.

$\xi_{J}^{\mathrm{AD}}$ in the optimized $\mathrm{W}(\mathrm{O}, \mathrm{N})$ samples $\mathrm{S} 4$ is determined to be $-42.2 \%$ and $-44.4 \%$ for thicknesses of 3.6 and 6 $\mathrm{nm}$, respectively. This is amongst the highest reported in the literature for PMA samples. The increased antidamping efficiency in $\mathrm{W}(\mathrm{O}, \mathrm{N})$ could be due to the tungsten being more amorphous than in the other samples, as can be inferred from the XRD spectra. $\xi_{J}^{\mathrm{FL}}$ for a thickness of $3.6 \mathrm{~nm}$ is found to be $-11.2 \%$, and for $6 \mathrm{~nm}$ it decreases to $-7.4 \%$.

Using a drift-diffusion model of spin-current generation by the spin Hall effect, we can estimate a spin diffusion length, $\lambda_{\mathrm{sf}}$, and an intrinsic or bulk spin Hall angle, $\theta_{\mathrm{SH}}^{\mathrm{eff}}$, from the relation $\xi_{j}^{\mathrm{AD}}=\theta_{\mathrm{SH}}^{\mathrm{eff}}\left[1-\operatorname{sech}\left(\mathrm{t}_{\mathrm{SOT}} / \lambda_{\mathrm{sf}}\right)\right][54]$. $\theta_{\mathrm{SH}}^{\text {eff }}$ and $\lambda_{\text {sf }}$ of samples S1, S2, and S3 are summarized in Table I. W(N) has the highest intrinsic spin Hall angle, of $-46.9 \pm 2.2 \%$, and its spin diffusion length is $1.8 \pm 0.1$ $\mathrm{nm}$. The lowest $\theta_{\mathrm{SH}}^{\mathrm{eff}}$, of $-36.6 \pm 5.6 \%$, is found in the $\mathrm{W}(\mathrm{O})$ samples, with an associated $\lambda_{\mathrm{sf}}$ of $2.3 \pm 0.4 \mathrm{~nm}$. $\theta_{\mathrm{SH}}^{\mathrm{eff}}$ and $\lambda_{\text {sf }}$ of the $\mathrm{W}$ in $\mathrm{S} 1$ are determined to be $-43 \pm 3.8 \%$ and $1.7 \pm 0.2 \mathrm{~nm}$, respectively, and these values are comparable to the values reported in the literature for $\mathrm{W}$ [33-35].

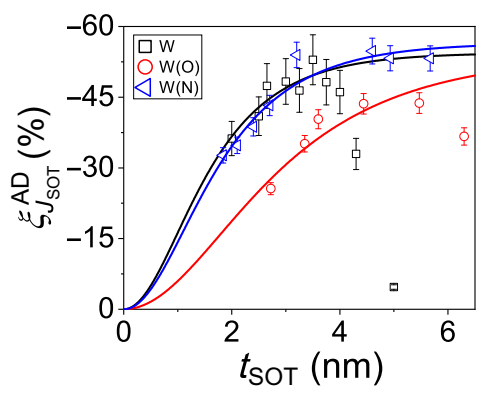

FIG. 5. $\xi_{J_{\mathrm{SOT}}}^{\mathrm{AD}}$ renormalized to the current flowing through the SOT layer, $I_{\text {SOT }}$. The solid lines are a fit of a drift-diffusion model to the data.
Thus far, the efficiency values mentioned are calculated from the total current $\left(I_{\text {total }}\right)$ applied; this current flows through all the conductive layers of the stack. Hence, assuming spin-current generation from the bulk spin Hall effect, we now recalculate $\xi_{J_{\mathrm{SOT}}}^{\mathrm{AD}}$ from the current flowing in the SOT layer $\left(I_{\mathrm{SOT}}\right)$. $I_{\mathrm{SOT}}$ is obtained by using the $\mathrm{W}$ and $\mathrm{Co}-\mathrm{Fe}-\mathrm{B}$ resistivities estimated from the linear dependence of the Hall-bar channel conductance on $t_{\text {SOT }}$.

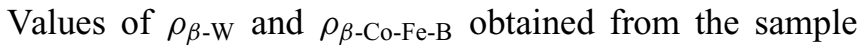
conductance are presented in Table I. The intrinsic spin Hall angle $\theta_{\mathrm{SH}}^{\text {eff }}$ and $\lambda_{\mathrm{sf}}$ estimated from the renormalized efficiencies, and the corresponding spin Hall conductivity $\sigma_{\mathrm{SH}} \approx \rho_{\beta-\mathrm{W}} \times \theta_{\mathrm{SH}}^{\text {eff }}\left(I_{\mathrm{SOT}}\right)$ are also tabulated.

The trends in the efficiencies are displayed in Fig. 5. After rectifying $\xi_{J}^{\mathrm{AD}}$ to $I_{\mathrm{SOT}}\left(\xi_{J_{\mathrm{SOT}}}^{\mathrm{AD}}\right)$, the trends become clear, and $\theta_{\mathrm{SH}}^{\text {eff }}$ is approximately the same in samples $\mathrm{S} 1-\mathrm{S} 3$, the only difference being that the spin diffusion length of $\mathrm{W}(\mathrm{O})$ is larger, $2 \pm 0.3 \mathrm{~nm}$, compared with $\mathrm{W}$ and $\mathrm{W}(\mathrm{N})$, in which $\lambda_{\text {sf }}$ is $1.1 \pm 0.1$ and $1.2 \pm 0.1 \mathrm{~nm}$, respectively. Thus, it is clear that the role of doping is mainly to extend the $\beta$ phase to thicker $t_{\mathrm{SOT}}$ and to minimize the formation of $\alpha-\mathrm{W}$ grains. Ignoring a possible interface contribution, the effective spin Hall angle appears to be an inherent property and therefore does not change considerably. Finally, the spin Hall conductivities of samples S1-S3 are larger than in previous reports [33]. The values of $\sigma_{\mathrm{SH}}$ for $\mathrm{S} 1$ and $\mathrm{S} 3$ are similar, and $\mathrm{W}(\mathrm{O})$ has the lowest value, of 3.1 $\mathrm{k}(\Omega \mathrm{m})^{-1}$.

\section{SPIN-ORBIT-TORQUE SWITCHING OF SOT-MRAM CELL WITH W AND W(O,N) AS SOT LAYERS}

As mentioned in the previous section, based on the information obtained from the doping study, we optimize the recipes for $\mathrm{W}(\mathrm{O}, \mathrm{N})$ - which has the highest SOT among the samples discussed in this study - for two thicknesses of interest for our SOT-MTJ development: 3.6 and $6 \mathrm{~nm}$. Only the 6-nm case is integrated into SOT-MRAM cells. After MTJ stack deposition and annealing at $300^{\circ} \mathrm{C}$, the TMR is evaluated by the current-in-plane tunneling method, and both stacks exhibit similar values to those of W-based stacks, of approximately $100 \%$. 
(a)
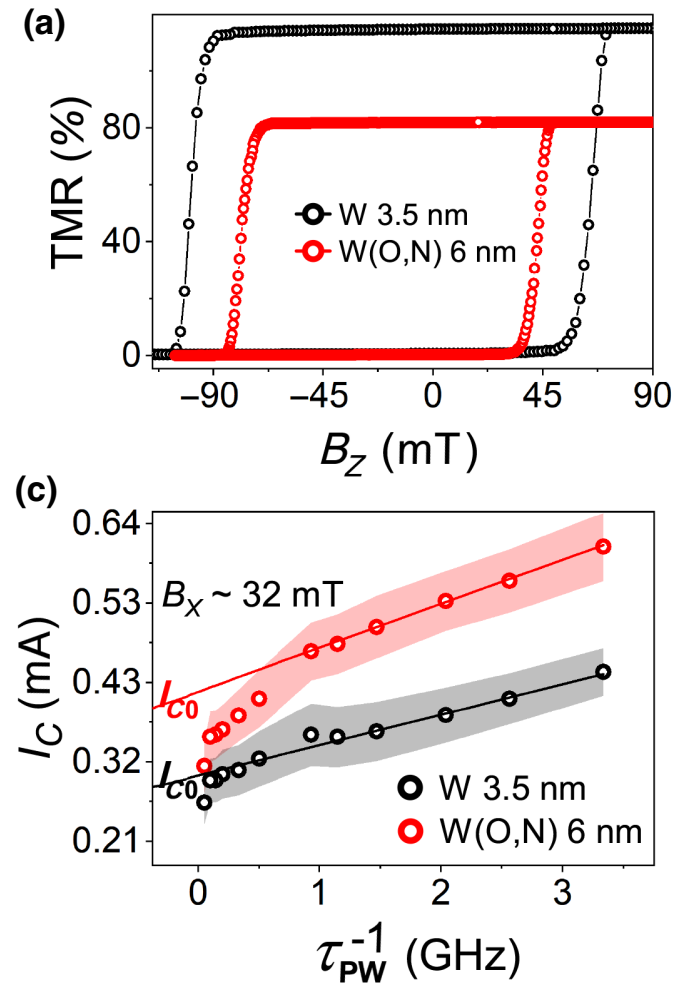

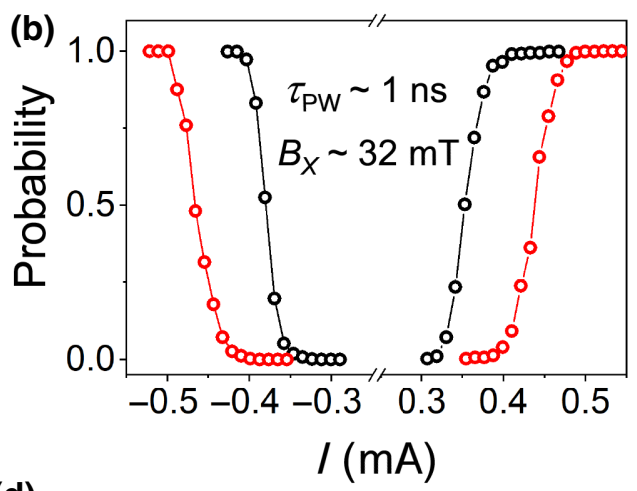

(d)

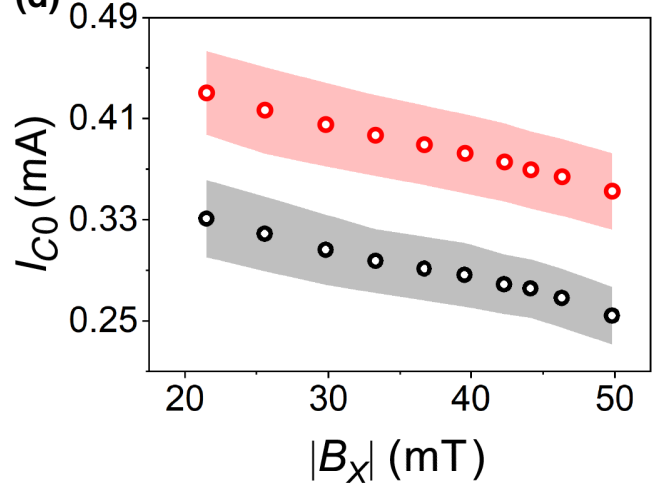

FIG. 6. (a) Hysteresis loops of the free layer of a PMTJ SOT-MRAM cell with $\mathrm{W}$ and $\mathrm{W}(\mathrm{O}, \mathrm{N})$ as SOT layers. (b) Electricalswitching (SOT) probability distribution for a $60-\mathrm{nm}$ MTJ with $\mathrm{W}$ and $\mathrm{W}(\mathrm{O}, \mathrm{N})$ as SOT-generating layers, as a function of SOT current $I$. The width of the SOT pulse used is $1 \mathrm{~ns}$. An in-plane field $\left(B_{X}\right)$ of $32 \mathrm{mT}$ is applied during the switching measurements. (c) Pulse-width dependence of threshold current $I_{C}$ for switching a 60-nm PMTJ by SOT, for $\mathrm{W}$ and $\mathrm{W}(\mathrm{O}, \mathrm{N})$ SOT layers. (d) Dependence of $I_{C 0}$ on inplane magnetic field $\left|B_{X}\right|$ for $\mathrm{W}$ and $\mathrm{W}(\mathrm{O}, \mathrm{N})$ SOT layers in a PMTJ SOT-MRAM cell.
To confirm the improvement of the switching properties in 6-nm $\mathrm{W}(\mathrm{O}, \mathrm{N})$ against standard $\mathrm{W}$ devices, 6-nm $\mathrm{W}(\mathrm{O}, \mathrm{N})$-based three-terminal SOT-MTJs are fabricated following the process flow described in Ref. [10]. The dimensions of devices reported here are of a $60-\mathrm{nm}$ diameter PMTJ sitting on a $160-\mathrm{nm}$-wide SOT track. Typical $R-H$ loops of the free layer are shown in Fig. 6(a). Both samples show similar values of the free-layer coercivity $\left(B_{c} \approx 75 \pm 5 \mathrm{mT}\right)$ and offset field $\left(B_{\text {off }} \approx-17 \pm 0.5 \mathrm{mT}\right)$; however, we note a significant drop in the TMR from $115 \%$ for $\mathrm{W}$ to $80 \%$ for $\mathrm{W}(\mathrm{O}, \mathrm{N})$, which differs from the blanket estimations. The reduction in the TMR could be an impact of device processing. Additionally, we estimate the thermal stability $(\Delta)$ and anisotropy $\left(B_{K}^{\text {eff }}\right)$ of the free layer using the probability distribution of $B_{S}$ [43], as detailed in Sec. II. The effective anisotropy $B_{K}^{\text {eff }}$ of a W $[\mathrm{W}(\mathrm{O}, \mathrm{N})]$ PMTJ is found to be $171.6 \pm 5.8 \mathrm{mT}[190.7 \pm 4.2 \mathrm{mT}]$, and the corresponding thermal stability is $47.1 \pm 1(39.3 \pm 0.8)$.

Next, we compare the SOT switching properties of optimized $\mathrm{W}(\mathrm{O}, \mathrm{N})$ with those of nondoped $\mathrm{W}$. To serve this purpose, electrical switching measurements are performed in the presence of an in-plane field $\left(B_{X}\right)$ along the direction of the current and an out-of-plane field equal and opposite to the offset field. The switching probability distribution $P_{\text {sw }}$ is determined from over 1000 switching events as a function of the current. The threshold current $I_{C}$, defined at $50 \%$ probability, is estimated from $P_{\mathrm{sw}}$ by fitting an exponential function [10]. Characteristic switching probability distributions for $\mathrm{W}$ and $\mathrm{W}(\mathrm{O}, \mathrm{N})$ at a pulse width $\tau_{\mathrm{PW}}=1$ ns and $B_{X} \approx 32 \mathrm{mT}$, for positive and negative currents, are shown in Fig. 6(b). In the following, we define $I_{C}$ as being the average of the anti-parallel to parallel and parallel to anti-parallel switching-current values to alleviate the problem of writing asymmetries induced by the referencelayer offset field. $I_{C}$ is then plotted as a function of the inverse of $\tau_{\mathrm{PW}}$, as presented in Fig. 6(c). As reported in Ref. [55], $I_{C}$ manifests two different regimes: until a 1-ns pulse width, switching is thermally assisted, but below 1 ns it is in the intrinsic regime and $I_{C}$ scales linearly with the frequency $1 / \tau_{\mathrm{PW}}$, i.e., $I_{C}=I_{C 0}+\left(Q / \tau_{\mathrm{PW}}\right)$. The intrinsic threshold current density for switching, $I_{C 0}$, is defined as the intercept of a linear fit to the intrinsic regime, and the slope $Q$ is related to the reversal mechanism (it is related to the nucleation energy and domain-wall propagation). We extract $I_{C 0}=0.31 \mathrm{~mA}$ for $6-\mathrm{nm} \mathrm{W}(\mathrm{O}, \mathrm{N})$ devices, which is approximately 1.33 times the value for the $\mathrm{W}$ devices $(0.232 \mathrm{~mA})$. When normalized to the same thickness of the SOT layer, $I_{C 0}$ for $\mathrm{W}(\mathrm{O}, \mathrm{N})$ is approximately $25 \%$ lower than for $\mathrm{W}$ (the $I_{C 0}$ ratio of 1.33 is less than the thickness ratio of the SOT layers, approximately 1.71 ). This is consistent with the observation in Sec. V, where the effective $\xi_{J}^{\mathrm{AD}}$ is found to be $25 \%$ larger in the $\mathrm{W}(\mathrm{O}, \mathrm{N})$ system. This shows that the dependence of the SOT efficiency in the trilayer stack is still reflected in the fully integrated SOTMRAM PMTJ cell. In contrast, the $Q$ factor is similar for both samples, which should be the case for very similar free-layer characteristics of both samples.

Finally, we measure $I_{C 0}$ as a function of the applied in-plane magnetic field $B_{X}$. To mitigate writing-current asymmetries induced by the offset field from the reference 
layer, we report in Fig. 6(d) the average $I_{C 0}$ obtained for positive and negative $B_{X}$ as a function of $\left|B_{X}\right|$. We observe that $I_{C 0}$ reduces monotonically with the strength of the in-plane magnetic field, as expected from Eq. (1). A linear fit of Eq. (1) to the $I_{C 0}$ - $\mathrm{Vs}-B_{X}$ data gives the spin Hall efficiency $\xi_{J}^{\mathrm{AD}}$ as the dependent parameter. We find that the antidamping SOT efficiencies of $\mathrm{W}$ and $\mathrm{W}(\mathrm{O}, \mathrm{N})$ are $-0.13 \pm 0.03$ and $-0.18 \pm 0.04$, respectively. These are significantly lower than the values reported in Sec. V (approximately -0.35 and -0.44 , respectively), quantified by the second-harmonic Hall-voltage technique on Hallbar devices, but consistently approximately $38 \%$ larger for the $\mathrm{W}(\mathrm{O}, \mathrm{N})$ system. This discrepancy may stem from Eq. (1), which is a simplified model derived from a macrospin model at $0 \mathrm{~K}$ that assumes only that the antidamping torque influences the magnetization reversal. In contrast, it has been shown that the reversal mechanisms are not macrospin, and that the increase in temperature due to the switching pulse, the fieldlike torque, and the Dzyaloshinskii-Moriya interaction impact reversal [56]. We therefore conclude that while Eq. (1) qualitatively describes SOT-mediated reversal, it cannot be used for quantitative estimation of $\xi_{J}^{\mathrm{AD}}$, showing the need to develop new techniques to quantify SOT efficiencies at the SOT-MRAM-cell level and more refined analytical models capturing the correct switching mechanisms.

\section{CONCLUSIONS}

In summary, our nondoped $\mathrm{W}$ undergoes a $\beta$-to- $\alpha$ phase transition around $4 \mathrm{~nm}$, resulting in a loss of PMA and SOT efficiency after the transition. Next, by doping tungsten (separately) with oxygen, denoted as $\mathrm{W}(\mathrm{O})$, or nitrogen, denoted as $\mathrm{W}(\mathrm{N})$, the $\beta$ phase is extended to thicker tungsten (more than $4 \mathrm{~nm}$ ). However, we find that Co-Fe-B loses PMA in the $\mathrm{W}(\mathrm{N})$ samples, and $M_{s}$ is reduced in the $\mathrm{W}(\mathrm{O})$ samples. The antidamping SOT efficiency in $\mathrm{W}(\mathrm{N})$ is found to be $-41.5 \%$, which is higher than that in $\mathrm{W}(\mathrm{O})$, $-32.3 \%$. Interestingly, in both nondoped $\mathrm{W}$ and doped tungsten samples $[\mathrm{W}(\mathrm{N}), \mathrm{W}(\mathrm{O})]$, the bulk intrinsic spin Hall efficiency is determined to be similar, approximately $-56 \%$, despite the variation in the spin diffusion length. This shows that the role of the dopants is to extend the nucleation of amorphous $\beta$-W to thicker tungsten and not to significantly influence the intrinsic spin Hall efficiency of tungsten.

Consequently, we prepare a bilayer lattice of nitrogenand oxygen-doped tungsten, $\mathrm{W}(\mathrm{O}, \mathrm{N})$, to make use of the best properties resulting from doping separately, such as improved PMA and AD-SOT efficiency. Further, in $\mathrm{W}(\mathrm{O}, \mathrm{N})$, we demonstrate that the critical thickness at which tungsten undergoes the $\beta$-to- $\alpha$ transition can be extended to greater than $10 \mathrm{~nm}$. Moreover, Co-Fe-B deposited on this optimized tungsten, $\mathrm{W}(\mathrm{O}, \mathrm{N})$, has PMA, a crucial requirement for application in PMTJ SOT
MRAMs. In $\mathrm{W}(\mathrm{O}, \mathrm{N})$, the antidamping SOT efficiency is $-44.4 \%$, which is higher than the value of $-35 \%$ in our nondoped $\mathrm{W}$ samples. Finally, we also integrate $\mathrm{W}(\mathrm{O}, \mathrm{N})(6 \mathrm{~nm})$ into SOT-MRAM devices and show that the intrinsic threshold current for switching the free layer is effectively reduced by $25 \%$ compared with nondoped $\mathrm{W}(3.5 \mathrm{~nm})$ devices. Thus, the SOT efficiency optimized at the level of simplified trilayer stacks is also reflected at the level of SOT-MRAM cells. Our work shows the possibility of further optimizing and engineering W-based SOT-MRAM technology toward application requirement targets.

\section{ACKNOWLEDGMENTS}

This work is supported by imec's Industrial Affiliation Program on MRAM devices. We thank Dr. Kaiming Cai for helpful discussions.

[1] Aurelien Manchon, Jakub Železnỳ, Ioan M. Miron, Tomas Jungwirth, Jairo Sinova, André Thiaville, Kevin Garello, and Pietro Gambardella, Current-induced spin-orbit torques in ferromagnetic and antiferromagnetic systems, Rev. Mod. Phys. 91, 035004 (2019).

[2] Ioan Mihai Miron, Kevin Garello, Gilles Gaudin, PierreJean Zermatten, Marius V. Costache, Stéphane Auffret, Sébastien Bandiera, Bernard Rodmacq, Alain Schuhl, and Pietro Gambardella, Perpendicular switching of a single ferromagnetic layer induced by in-plane current injection, Nature 476, 189 (2011).

[3] Luqiao Liu, Chi-Feng Pai, Y. Li, H. W. Tseng, D. C. Ralph, and R. A. Buhrman, Spin-torque switching with the giant spin Hall effect of tantalum, Science 336, 555 (2012).

[4] Murat Cubukcu, Olivier Boulle, Marc Drouard, Kevin Garello, Can Onur Avci, Ioan Mihai Miron, Juergen Langer, Berthold Ocker, Pietro Gambardella, and Gilles Gaudin, Spin-orbit torque magnetization switching of a three-terminal perpendicular magnetic tunnel junction, Appl. Phys. Lett. 104, 042406 (2014).

[5] Murat Cubukcu, Olivier Boulle, Nikolaï Mikuszeit, Claire Hamelin, Thomas Brächer, Nathalie Lamard, Marie-Claire Cyrille, Liliana Buda-Prejbeanu, Kevin Garello, and Ioan Mihai Miron et al., Ultra-fast perpendicular spin-orbit torque mram, IEEE Trans. Magn. 54, 1 (2018).

[6] Sk Ziaur Rahaman, I.-Jung Wang, Tian-Yue Chen, ChiFeng Pai, Ding-Yeong Wang, Jeng-Hua Wei, Hsin-Han Lee, Yu-Chen Hsin, Yao-Jen Chang, and Shan-Yi Yang et al., Pulse-width and temperature effect on the switching behavior of an etch-stop-on-MgO-barrier spin-orbit torque mram cell, IEEE Electron Device Lett. 39, 1306 (2018).

[7] Sk Ziaur Rahaman, I.-Jung Wang, Ding-Yeong Wang, ChiFeng Pai, Yu-Chen Hsin, Shan-Yi Yang, Hsin-Han Lee, Yao-Jen Chang, Yi-Ching Kuo, and Yi-Hui Su et al., Sizedependent switching properties of spin-orbit torque mram with manufacturing-friendly 8 -inch wafer-level uniformity, IEEE J. Electron Devices Soc. 8, 163 (2020). 
[8] H. Honjo, T. V. A. Nguyen, T. Watanabe, T. Nasuno, C. Zhang, T. Tanigawa, S. Miura, H. Inoue, M. Niwa, and T. Yoshiduka et al., in 2019 IEEE International Electron Devices Meeting (IEDM) (IEEE, San Francisco, CA, USA, 2019), p. 28.

[9] Noriyuki Sato, Gary A. Allen, William P. Benson, Benjamin Buford, Atreyee Chakraborty, Michael Christenson, Tanay A. Gosavi, Philip E. Heil, Nafees A. Kabir, and Brian J. Krist et al., in 2020 IEEE Symposium on VLSI Technology (IEEE, Honolulu, HI, USA, 2020), p. 1.

[10] K. Garello, F. Yasin, S. Couet, L. Souriau, J. Swerts, S. Rao, S. Van Beek, W. Kim, E. Liu, and S. Kundu, Sot-mram $300 \mathrm{~mm}$ integration for low power and ultrafast embedded memories, 2018 IEEE Symposium on VLSI Circuits (IEEE, 2018), p. 81.

[11] Kevin Garello, Farrukh Yasin, and Gouri Sankar Kar, in 2019 IEEE 11th International Memory Workshop (IMW) (2019 IEEE 11th International Memory Workshop (IMW), 2019), p. 1.

[12] Stuart Parkin and See-Hun Yang, Memory on the racetrack, Nat. Nanotechnol. 10, 195 (2015).

[13] Giacomo Talmelli, Florin Ciubotaru, Kevin Garello, Xiao Sun, Marc Heyns, Iuliana P. Radu, Christoph Adelmann, and Thibaut Devolder, Spin-Wave Emission by Spin-OrbitTorque Antennas, Phys. Rev. Appl. 10, 044060 (2018).

[14] Himanshu Fulara, Mohammad Zahedinejad, Roman Khymyn, Ahmad Awad, Shreyas Muralidhar, Mykola Dvornik, and Johan Åkerman, Spin-orbit-torque driven propagating spin waves, arXiv:1904.06945 (2019).

[15] Abhronil Sengupta, Sri Harsha Choday, Yusung Kim, and Kaushik Roy, Spin orbit torque based electronic neuron, Appl. Phys. Lett. 106, 143701 (2015).

[16] Jonas Dpevenspeck and Kevin Garello et al., Sot-mram based analog in-memory computing for dnn inference, IEE Symposium on VLSI, JFS4.1 (2020).

[17] Saima A. Siddiqui, Sumit Dutta, Astera Tang, Luqiao Liu, Caroline A. Ross, and Marc A. Baldo, Magnetic domain wall based synaptic and activation function generator for neuromorphic accelerators, Nano. Lett. 20, 1033 (2019).

[18] Can Onur Avci, Kevin Garello, Corneliu Nistor, Sylvie Godey, Belén Ballesteros, Aitor Mugarza, Alessandro Barla, Manuel Valvidares, Eric Pellegrin, Abhijit Ghosh, Ioan Mihai Miron, Olivier Boulle, S. Auffret, Gilles Gaudin, and Pietro Gambardella, Fieldlike and antidamping spin-orbit torques in as-grown and annealed $\mathrm{Ta} / \mathrm{CoFeB} / \mathrm{MgO}$ layers, Phys. Rev. B 89, 214419 (2014).

[19] A. Van den Brink, S. Cosemans, S. Cornelissen, M. Manfrini, A. Vaysset, W. Van Roy, T. Min, H. J. M. Swagten, and B. Koopmans, Spin-Hall-assisted magnetic random access memory, Appl. Phys. Lett. 104, 012403 (2014).

[20] Young-Wan Oh, Seung-Heon Chris Baek, Y. M. Kim, Hae Yeon Lee, Kyeong-Dong Lee, Chang-Geun Yang, EunSang Park, Ki-Seung Lee, Kyoung-Whan Kim, Gyungchoon Go, Jong-Ryul Jeong, Byoung-Chul Min, HyunWoo Lee, Kyung-Jin Lee, and Byong-Guk Park, Field-free switching of perpendicular magnetization through spinorbit torque in antiferromagnet/ferromagnet/oxide structures, Nat. Nanotechnol. 11, 878 (2016).

[21] Yong-Chang Lau, Davide Betto, Karsten Rode, J. M. D. Coey, and Plamen Stamenov, Spin-orbit torque switching without an external field using interlayer exchange coupling, Nat. Nanotechnol. 11, 758 (2016).

[22] Long You, OukJae Lee, Debanjan Bhowmik, Dominic Labanowski, Jeongmin Hong, Jeffrey Bokor, and Sayeef Salahuddin, Switching of perpendicularly polarized nanomagnets with spin orbit torque without an external magnetic field by engineering a tilted anisotropy, Proc. National Acad. Sci. 112, 10310 (2015).

[23] Mengxing Wang, Wenlong Cai, Daoqian Zhu, Zhaohao Wang, Jimmy Kan, Zhengyang Zhao, Kaihua Cao, Zilu Wang, Youguang Zhang, and Tianrui Zhang et al., Fieldfree switching of a perpendicular magnetic tunnel junction through the interplay of spin-orbit and spin-transfer torques, Nat. Electron. 1, 582 (2018).

[24] K. Garello, F. Yasin, H. Hody, S. Couet, L. Souriau, S. H. Sharifi, J. Swerts, R. Carpenter, S. Rao, and W. Kim et al., in 2019 Symposium on VLSI Circuits (2019 Symposium on VLSI Circuits, 2019), p. T194.

[25] Eva Grimaldi, Viola Krizakova, Giacomo Sala, Farrukh Yasin, Sébastien Couet, Gouri Sankar Kar, Kevin Garello, and Pietro Gambardella, Single-shot dynamics of spin-orbit torque and spin transfer torque switching in three-terminal magnetic tunnel junctions, Nat. Nanotechnol. 15, 111 (2020).

[26] Ki-Seung Lee, Seo-Won Lee, Byoung-Chul Min, and Kyung-Jin Lee, Threshold current for switching of a perpendicular magnetic layer induced by spin Hall effect, Appl. Phys. Lett. 102, 112410 (2013).

[27] Pietro Gambardella and Ioan Mihai Miron, Current-induced spin-orbit torques, Philos. Trans. R. Soc. London A: Math. Phys. Eng. Sci. 369, 3175 (2011).

[28] Aurelien Manchon, Hyun Cheol Koo, Junsaku Nitta, S. M. Frolov, and R. A. Duine, New perspectives for Rashba spin-orbit coupling, Nat. Mater. 14, 871 (2015).

[29] Lijun Zhu and R. A. Buhrman, Maximizing Spin-OrbitTorque Efficiency of Pt/Ti Multilayers: Trade-Off between Intrinsic Spin Hall Conductivity and Carrier Lifetime, Phys. Rev. Appl. 12, 051002 (2019).

[30] A. R. Mellnik, J. S. Lee, A Richardella, J. L. Grab, P. J. Mintun, Mark H. Fischer, Abolhassan Vaezi, Aurelien Manchon, E.-A. Kim, and Nitin Samarth et al., Spintransfer torque generated by a topological insulator, Nature 511, 449 (2014).

[31] Yi Wang, Dapeng Zhu, Yang Wu, Yumeng Yang, Jiawei Yu, Rajagopalan Ramaswamy, Rahul Mishra, Shuyuan Shi, Mehrdad Elyasi, and Kie-Leong Teo et al., Room temperature magnetization switching in topological insulatorferromagnet heterostructures by spin-orbit torques, Nat. Commun. 8, 1 (2017).

[32] Chi-Feng Pai, Luqiao Liu, Y. Li, H. W. Tseng, D. C. Ralph, and R. A. Buhrman, Spin transfer torque devices utilizing the giant spin Hall effect of tungsten, Appl. Phys. Lett. 101, 122404 (2012).

[33] Jun Liu, Tadakatsu Ohkubo, Seiji Mitani, Kazuhiro Hono, and Masamitsu Hayashi, Correlation between the spin Hall angle and the structural phases of early $5 \mathrm{~d}$ transition metals, Appl. Phys. Lett. 107, 232408 (2015).

[34] Yutaro Takeuchi, Chaoliang Zhang, Atsushi Okada, Hideo Sato, Shunsuke Fukami, and Hideo Ohno, Spin-orbit torques in high-resistivity-W/CoFeB/MgO, Appl. Phys. Lett. 112, 192408 (2018). 
[35] Qiang Hao and Gang Xiao, Giant Spin Hall Effect and Switching Induced by Spin-Transfer Torque in a W/Co 40 $\mathrm{Fe} 40$ B 20/MgO Structure with Perpendicular Magnetic Anisotropy, Phys. Rev. Appl. 3, 034009 (2015).

[36] Qiang Hao, Wenzhe Chen, and Gang Xiao, Beta $(\beta)$ tungsten thin films: Structure, electron transport, and giant spin Hall effect, Appl. Phys. Lett. 106, 182403 (2015).

[37] Jiaxing Liu and Katayun Barmak, Topologically closepacked phases: Deposition and formation mechanism of metastable $\beta$-w in thin films, Acta Mater. 104, 223 (2016).

[38] Avyaya J. Narasimham, Manasa Medikonda, Akitomo Matsubayashi, Prasanna Khare, Hyuncher Chong, Richard J. Matyi, Alain Diebold, and Vincent P. LaBella, Fabrication of 5-20 nm thick $\beta$-w films, AIP Adv. 4, 117139 (2014).

[39] Avyaya J. Narasimham, Avery Green, Richard J. Matyi, Prasanna Khare, Tuan Vo, and Alain Diebold, and Vincent P. LaBella, Pulsed-n 2 assisted growth of 5-20 nm thick $\beta$-w films, AIP Adv. 5, 117107 (2015).

[40] Kevin Garello, Ioan Mihai Miron, Can Onur Avci, Frank Freimuth, Yuriy Mokrousov, Stefan Blügel, Stéphane Auffret, Olivier Boulle, Gilles Gaudin, and Pietro Gambardella, Symmetry and magnitude of spin-orbit torques in ferromagnetic heterostructures, Nat. Nanotechnol. 8, 587 (2013).

[41] Can Onur Avci, Kevin Garello, Mihai Gabureac, Abhijit Ghosh, Andreas Fuhrer, Santos F. Alvarado, and Pietro Gambardella, Interplay of spin-orbit torque and thermoelectric effects in ferromagnet/normal-metal bilayers, Phys. Rev. B 90, 224427 (2014).

[42] Masamitsu Hayashi, Junyeon Kim, Michihiko Yamanouchi, and Hideo Ohno, Quantitative characterization of the spinorbit torque using harmonic Hall voltage measurements, Phys. Rev. B 89, 144425 (2014).

[43] Luc Thomas, Guenole Jan, Jian Zhu, Huanlong Liu, YuanJen Lee, Son Le, Ru-Ying Tong, Keyu Pi, Yu-Jen Wang, and Dongna Shen et al., Perpendicular spin transfer torque magnetic random access memories with high spin torque efficiency and thermal stability for embedded applications, J. Appl. Phys. 115, 172615 (2014).

[44] Dooho Choi, Bincheng Wang, Suk Chung, Xuan Liu, Amith Darbal, Adam Wise, Noel T. Nuhfer, Katayun Barmak, Andrew P. Warren, and Kevin R. Coffey et al., Phase, grain structure, stress, and resistivity of sputter-deposited tungsten films, J. Vac. Sci. Technol. A: Vac. Surf. Films 29, 051512 (2011).

[45] Ananya Chattaraj, Mohammad Balal, Ashok Kumar Yadav, Sudipta Roy Barman, Anil Kumar Sinha, Shambhu Nath Jha, Sebastien Joulie, Virginie Serin, Alain Claverie, and Vijay Kumar et al., Unravelling oxygen driven $\alpha$ to $\beta$ phase transformation in tungsten, Sci. Rep. 10, 1 (2020).
[46] Kai-Uwe Demasius, Timothy Phung, Weifeng Zhang, Brian P. Hughes, See-Hun Yang, Andrew Kellock, Wei Han, Aakash Pushp, and Stuart S. P. Parkin, Enhanced spinorbit torques by oxygen incorporation in tungsten films, Nat. Commun. 7, 10644 (2016).

[47] G. S. Kar, W. Kim, T. Tahmasebi, J. Swerts, S. Mertens, N. Heylen, and T. Min, in 2014 IEEE International Electron Devices Meeting (2014 IEEE International Electron Devices Meeting, 2014), p. 19.

[48] K. Hasegawa, Y. Hibino, M. Suzuki, T. Koyama, and D. Chiba, Enhancement of spin-orbit torque by inserting coo $\mathrm{X}$ layer into co/pt interface, Phys. Rev. B 98, 020405 (2018).

[49] Léa Cuchet, Bernard Rodmacq, Stéphane Auffret, Ricardo C. Sousa, Ioan L. Prejbeanu, and Bernard Dieny, Perpendicular magnetic tunnel junctions with double barrier and single or synthetic antiferromagnetic storage layer, J. Appl. Phys. 117, 233901 (2015).

[50] Sebastien Couet, Thibaut Devolder, Johan Swerts, Sofie Mertens, Tsann Lin, Enlong Liu, Sven Van Elshocht, and G. Sankar Kar, Impact of Ta and W-based spacers in double MgO STT-MRAM free layers on perpendicular anisotropy and damping, Appl. Phys. Lett. 111, 152406 (2017).

[51] Bernard Rodmacq, Aurélien Manchon, Clarisse Ducruet, Stéphane Auffret, and Bernard Dieny, Influence of thermal annealing on the perpendicular magnetic anisotropy of pt/co/alox trilayers, Phys. Rev. B 79, 024423 (2009).

[52] B. Dieny and M. Chshiev, Perpendicular magnetic anisotropy at transition metal/oxide interfaces and applications, Rev. Mod. Phys. 89, 025008 (2017).

[53] Sucheta Mondal, Samiran Choudhury, Neha Jha, Arnab Ganguly, Jaivardhan Sinha, and Anjan Barman, All-optical detection of the spin Hall angle in $\mathrm{W} / \mathrm{CoFeB} / \mathrm{SiO} 2$ heterostructures with varying thickness of the tungsten layer, Phys. Rev. B 96, 054414 (2017).

[54] Luqiao Liu, Takahiro Moriyama, D. C. Ralph, and R. A. Buhrman, Spin-Torque Ferromagnetic Resonance Induced by the Spin Hall Effect, Phys. Rev. Lett. 106, 036601 (2011).

[55] Kevin Garello, Can Onur Avci, Ioan Mihai Miron, Manuel Baumgartner, Abhijit Ghosh, S. Auffret, Olivier Boulle, Gilles Gaudin, and Pietro Gambardella, Ultrafast magnetization switching by spin-orbit torques, Appl. Phys. Lett. 105, 212402 (2014).

[56] N. Mikuszeit, O. Boulle, I. M. Miron, K. Garello, P. Gambardella, G. Gaudin, and L. D. Buda-Prejbeanu, Spin-orbit torque driven chiral magnetization reversal in ultrathin nanostructures, Phys. Rev. B 92, 144424 (2015).

Correction: The fourth affiliation contained an error and has been set right. 